\title{
Estudo da logística de distribuição física de um laticínio utilizando lógica fuzzy
}

\author{
Alysson Vinícius Neves dos Santos ${ }^{\mathrm{a} *}$, Leonardo Bonato Felix ${ }^{\mathrm{b}}$, José Geraldo Vidal Vieira ${ }^{\mathrm{c}}$ \\ a*alysson.santos@ufv.br, UFV, Brasil \\ bleobonato@ufv.br, UFV, Brasil \\ cjose-vidal@ufscar.br, UFSCar, Brasil
}

\begin{abstract}
Resumo
Este estudo versa sobre a viabilidade da aplicação da lógica fuzzy para auxiliar o gerente de uma indústria de laticínios na tomada de decisão na área de logística de distribuição física. 0 estudo de caso realizado numa indústria de laticínios de pequeno porte em Minas Gerais identificou que, a cada novo pedido solicitado via internet, o profissional de logística manipula ao mesmo tempo um grande número de informações e decide, com base na sua experiência, a melhor forma de distribuição desses pedidos. A proposta deste trabalho é apresentar um modelo fuzzy como uma alternativa de apoio aos métodos tradicionais de processamento de pedidos e tomada de decisão logística. Os resultados permitiram concluir que o modelo foi transcrito de forma adequada ao problema proposto ao apresentar as mesmas decisões tomadas pelo especialista e que, se observados os pontos fracos detectados, este modelo pode se constituir em importante ferramenta para a competitividade das indústrias.
\end{abstract}

Palavras-chave

Distribuição física. lógica fuzzy. Indústria de laticínio.

\section{Introdução}

Em todos os setores de atividades industriais, a importância da otimização da produtividade e eficiência dos processos e a melhoria na qualidade dos serviços prestados são cada vez mais crescentes, motivadas pela necessidade do aumento da competitividade em um mercado globalizado. 0 setor agroindustrial, ramo da indústria estudada neste trabalho, é bastante dependente dos processos logísticos de distribuição física, por lidar exclusivamente com produtos perecíveis.

A distribuição física compreende os processos de estocagem, transporte, controle, intercâmbio de dados e fluxo financeiro, que permitem transferir produtos desde o fabricante até o consumidor final. Ela tem como objetivo garantir que o produto certo seja entregue ao consumidor final, no lugar correto, no momento exato e com o nível de serviços desejado pelo cliente, buscando minimizar os custos dos serviços logísticos.

No estudo de caso deste trabalho foi identificado que um empecilho para a tomada de decisões em distribuição física na indústria é o volume de informações no processamento de pedidos que precisam ser analisadas simultaneamente, consumindo tempo e, consequentemente, incorrendo custos para a indústria.

Nesse contexto, ferramentas de inteligência artificial (IA) surgem como alternativas de apoio aos métodos tradicionais de processamento de pedidos e tomada de decisão em transportes, atividades-chave da logística de distribuição. A construção de uma metodologia de apoio à decisão através do uso de técnicas de IA com o intuito de viabilizar e facilitar o planejamento e controle das atividades logísticas é interessante não só do ponto de vista de gerenciamento do sistema, mas também da verificação da aplicabilidade dessas técnicas em relação ao problema proposto.

Assim, este trabalho propõe uma metodologia baseada em lógica fuzzy para auxiliar o tomador de decisão na escolha da melhor estratégia de distribuição 
dos produtos a partir do laticínio, tendo como foco a agilização dos processos de distribuição e a redução de custos logísticos.

\section{Revisão bibliográfica}

\subsection{Logística de distribuição física}

Segundo o Conselho de Profissionais da Gestão da Cadeia de Suprimentos*, logística é a parte da gestão da cadeia de suprimentos que planeja, implementa e controla o fluxo eficiente e eficaz (direto ou reverso) de mercadorias, serviços e informações relativas desde o ponto de origem até o ponto de consumo com o propósito de atender às exigências dos clientes (COUNCIL..., 2007).

A logística de distribuição física opera em um movimento à jusante da cadeia de suprimentos e se refere ao desempenho de todas as funções exigidas para ligar produtores a clientes finais. Bowersox, Closs e Cooper (2006) identificam quatro participantes do canal de distribuição: os fabricantes, atacadistas, varejistas e consumidores.

Com relação à estrutura, a distribuição física pode ser direta ou indireta. Na distribuição direta, o fabricante estrutura sua rede de transporte de forma que todas as entregas cheguem diretamente ao consumidor, sem envolvimento intermediário. Já na distribuição indireta, o produto passa por alguns intermediários (e.g., atacadistas e varejistas) antes de chegar ao consumidor, ou usuário final (BOWERSOX; CLOSS; COOPER, 2006; CHOPRA; MEINDL, 2003).

Bowersox, Closs e Cooper (2006) ainda classificam a distribuição física com relação à cobertura de mercado, podendo ser intensiva, seletiva ou exclusiva. A distribuição intensiva se dá quando a colocação do produto é feita em quantos pontos de venda ou lugares for possivel. Na distribuição seletiva a colocação de um produto é feita em um número mais limitado de pontos de venda. Por fim, a distribuição exclusiva é o oposto da distribuição intensiva e envolve a colocação de um produto em apenas um ponto de venda, em cada área geográfica.

As atividades que compõem a logística de distribuição podem variar de acordo com as empresas, mas as atividades-chave (i.e., aquelas que estão no circuito "crítico" do canal de distribuição física imediato de uma empresa) são as seguintes (BALLOU, 2006):

- Serviços ao cliente padronizados;

- Transporte;

- Gerência de estoques; e

* Tradução nossa para "Council of Supply Chain Management Professionals".
- Fluxos de informação e processamento de pedidos.

Os padrões dos serviços aos clientes definem a qualidade dos serviços, bem como o índice de agilidade com os quais o sistema logístico deve reagir. Ballou (2006) ressalta que os custos logísticos aumentam proporcionalmente ao nível de serviço oferecido.

0 transporte, de acordo com Gurgel (2000), é um elo essencial entre a expedição da empresa e o cliente. Em termos de distribuição física, o transporte consiste no movimento do produto entre a unidade produtiva e o cliente. Para Chopra e Meindl (2003), o transporte exerce um papel crucial na logística de distribuição uma vez que os produtos raramente são fabricados e consumidos no mesmo local.

Ballou (2006) lista cinco modais básicos de transporte: ferroviário, rodoviário, aéreo, aquaviário e dutovias. Pode-se utilizar um desses meios ou então uma combinação de dois ou mais modais, o transporte intermodal. 0 transporte intermodal tira vantagem das economias inerentes a cada modal e, com isso, oferece um serviço integrado a um custo total mais baixo (CHOPRA; MEINDL, 2003; BOWERSOX; CLOSS; COOPER, 2006).

A gerência de estoques e o manuseio de produtos também são componentes essenciais do conjunto de atividades logísticas, visto que Ballou (2006) estima que essas atividades sejam responsáveis por até $20 \%$ dos custos de distribuição física da empresa. A redução de custos pode ser obtida através de algumas atividades, como, por exemplo, a consolidação de cargas, onde o armazém recebe os materiais de diferentes fontes; o sortimento, onde a carga é reconfigurada enquanto ela é transportada da origem para o destino; e o adiamento, onde o armazém pode adiar o compromisso da configuração final de um produto ao completar o acondicionamento, a etiquetagem e as produções leves.

0 processamento de pedidos é a atividade-chave final. É representado por uma variedade de atividades, entre elas a preparação, transmissão, recebimento e expedição do produto e relatório da situação de pedido. Apesar de seus custos serem normalmente menores em relação aos do transporte ou aos de manutenção de estoque, o processamento de pedidos é um elemento importante na determinação do tempo total da entrega de mercadorias ou serviços a um cliente. Essa atividade desencadeia a movimentação dos produtos e o serviço de entrega (BALLOU, 2006).

\subsection{Lógica fuzzy}

A lógica fuzzy (ou lógica nebulosa) foi desenvolvida a partir de 1965 com os trabalhos do professor Lotfi Zadeh, para representar o conhecimento incerto ou impreciso (SANDRI; CORREA, 1999). 
Formalmente, um conjunto fuzzy $A$ pode ser expresso por (TSOUKALAS; UHRIG, 1997 apud FUJIMOTO, 2005):

$$
A=\left\{x, \mu_{A}(x) / x \in X\right\}
$$

Onde $X$ é o universo de discurso ao qual o conjunto pertence, $x$ um componente do conjunto fuzzy, e $\mu_{A}$ a função de pertinência. 0 universo de discurso indica o domínio em que o modelamento do sistema fuzzy é válido.

A função de pertinência $\mu_{A}$ indica o grau de pertinência (ou compatibilidade) entre $x$ e o conceito expresso por $A$ (SANDRI; CORREA, 1999):

- $\mu_{A}(x)=1$ indica que $x$ é completamente compatível com $A$;

- $\mu_{A}(x)=0$ indica que $x$ é completamente incompatível com $A$; e

- $0<\mu_{A}(x)<1$ indica que (x) é parcialmente compatível $\operatorname{com} A$, com grau $\mu_{A}(x)$.

A lógica fuzzy pode ser comparada com a lógica clássica (ou booleana), que trata com a definição clássica de conjuntos. Nessa definição, um certo valor pertence ou não a um determinado conjunto, sendo representado pelos valores 0 e 1 , respectivamente. No caso da lógica fuzzy, um certo valor pode pertencer a um determinado conjunto com um certo grau de pertinência $\mu_{A}(x)$.

Ainda de modo análogo à lógica clássica, na lógica fuzzy existe a necessidade de proceder às operações de união, interseção e complemento; ou, na nomenclatura da lógica booleana, E, OU e NEGAÇÃO (CHEN et al., 1996 apud FUJIMOTO, 2005). 0 análogo ao operador E na lógica fuzzyé conhecido como Norma-T, sendo que esse operador define a interseção entre dois conjuntos fuzzy (Equação 2). 0 análogo ao operador OU é chamado Conorma-T, e esse operador define a união entre dois conjuntos fuzzy (Equação 3):

$\mu_{A \cap B}=\min \left(\mu_{A}, \mu_{B}\right)$

$\mu_{A \cap B}=\max \left(\mu_{A}, \mu_{B}\right)$

onde $\mu_{A}$ e $\mu_{B}$ são as respectivas funções de pertinência dos conjuntos fuzzy $A$ e $B$.

0 análogo ao operador NEGAÇÃO é definido na Equação 4. Esse operador define o complemento de um conjunto fuzzy.

$\mu=1-\mu_{A}$

onde $\mu_{A}$ é a função de pertinência do conjunto fuzzy $A$.
0 mapeamento do conhecimento a respeito de um processo através de regras fuzzy do tipo SE-ENTÃO é possível através dos sistemas de inferência fuzzy, que pode ser dividido em três etapas: fuzzificação; regras e inferências; e defuzzificação, como mostra a Figura 1:

$\mathrm{Na}$ fuzzificação as entradas não fuzzy ou determinísticas são apresentadas ao sistema por intermédio de medições ou observações de dados, os quais são considerados como sendo o conjunto de dados de entrada do sistema. Nessa fase também ocorre a ativação das regras fuzzy relevantes para um dado sistema.

As regras são elementos da base de conhecimento e definem a estratégia de controle do sistema. Podem ser fornecidas por especialistas, com base em seu conhecimento a respeito do processo que se deseja analisar, em forma de sentenças linguísticas, e se constituem em aspecto fundamental no desempenho de um sistema de inferência fuzzy. Dessa forma, o sistema de inferência fuzzy terá um desempenho confiável e satisfatório somente se as regras expressarem o comportamento do sistema de forma fiel e consistente. Os métodos de extração de regras podem partir também de dados numéricos. Esses métodos são particularmente úteis em aplicações onde haja disponível um conjunto de dados numéricos que refletem o comportamento entrada/saída do sistema.

No processo de inferência ocorrem as operações com os conjuntos fuzzy. Um aspecto importante é a definição dos conjuntos fuzzy correspondentes às variáveis de entrada e às de saída, pois o desempenho do sistema de inferência dependerá do número de conjuntos e de sua forma adotada. É possível efetuar uma sintonia manual das funções de pertinências dos conjuntos, mas é mais comum empregarem-se métodos automáticos. A integração entre sistemas de inferências fuzzy e redes neurais artificiais tem se mostrado adequada para a sintonização das funções de pertinências, assim como para a geração automática de regras (SILVA, 2007).

Após o processo de inferência, tem-se o processo de defuzzificação que, de posse do conjunto fuzzy de saída adquirido através do processo de inferência, é

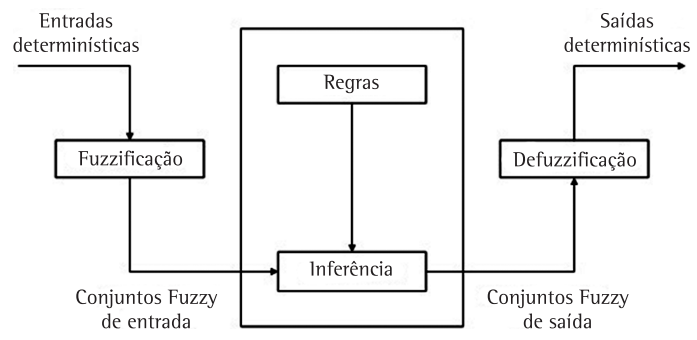

Figura 1. Diagrama do processo de inferência fuzzy. Fonte: adaptado de Silva (2007). 
responsável pela interpretação dessa informação para saídas precisas (dados não fuzzy). Isso se faz necessário, já que em aplicações práticas são requeridos valores não fuzzy. A técnica mais usual para defuzzificação é a do centroide, que utiliza a soma (ou integral) dos valores de pertinência multiplicados pelos valores das variáveis, dividindo esse valor final pela área total do conjunto (fuzzy) a ser defuzzificado (OLIVEIRA JUNIOR et al., 2007).

0 objetivo da lógica fuzzy é estudar os princípios formais do raciocínio aproximado, fazendo com que as decisões tomadas pela máquina se aproximem cada vez mais das decisões humanas (OLIVEIRA JUNIOR et al., 2007; GUIMARÃES et al., 2002 apud CAMARGOS, 2002). 0 enfoque fuzzy aliado à tecnologia possui grande valor prático, tornando possível a inclusão da experiência de especialistas em controles computadorizados e permitindo decisões em problemas complexos, como será apresentado neste trabalho.

\section{Materiais e métodos}

0 estudo de caso realizado em uma indústria de laticínios possibilitou um amplo e detalhado conhecimento das atividades logísticas dessa indústria, bem como a definição do escopo deste trabalho, que se limitou ao processamento de pedidos de clientes via internet e à forma como esses pedidos são distribuídos.

A partir de entrevistas com funcionários dessa indústria, foi levantada uma amostra composta de vinte exemplos de pedidos feitos via internet no ano de 2008 e as respectivas formas de distribuição desses pedidos. A coleta e análise dos dados se referiram à distância entre a indústria e o cliente (em km); ao valor total da nota fiscal (em R\$); à quantidade solicitada, em termos do peso total do pedido (em kg); à urgência ou não do pedido; e à forma de distribuição do pedido (entre as três opções disponíveis - transportadora 1, transportadora 2 e correios).

Para facilitar a modelagem do problema em lógica fuzzy, utilizou-se a Fuzzy Logic Toolbox ${ }^{T M}$ do software MATLAB ${ }^{\circledR}$. Os passos seguidos para a formulação do modelo de inferência fuzzy do tipo Mamdani foram (THE MATHWORKS, 2008; OLIVEIRA JUNIOR et al., 2007):

1. Elaboração das regras fuzzy - o modelo conta com nove regras, que foram criadas a partir da experiência do funcionário da indústria especialista em logística e da análise numérica das variáveis dos exemplos levantados;

2. Fuzzificação das entradas e definição das saídas: o modelo possui três entradas (peso, distância e urgência) e três saídas (transportadora 1, transportadora 2 e correios). Os parâmetros das funções de pertinência associadas a cada variável também foram especificados;

3. Ajuste das funções de inferência fuzzye método de defuzzificação;

4. Verificação do comportamento do modelo definido através da comparação com os exemplos levantados;

5. Validação do modelo, onde dez novos exemplos de pedidos via internet foram criados e fornecidos tanto ao sistema fuzzy quanto ao funcionário especialista em logística da indústria. A forma de distribuição escolhida pelo funcionário foi comparada com a resposta fornecida pelo sistema fuzzy.

\section{Resultados e discussões}

A indústria de laticínios onde o estudo de caso foi realizado se localiza na Zona da Mata de Minas Gerais e fabrica vários produtos derivados de leite, como iogurte, requeijão, manteiga, doce de leite, entre outros. A distribuição física é direta e a cobertura de mercado é classificada como intensiva, atendendo várias cidades de Minas Gerais e de outros estados, como Paraná, Espírito Santo, São Paulo e Rio de Janeiro. A indústria utiliza como forma exclusiva de transporte o modal rodoviário, com um caminhão próprio, oito caminhões de representantes terceirizados e ainda conta com os serviços de caminhões de duas transportadoras contratadas (aqui chamadas de transportadoras 1 e 2). 0 sistema de distribuição dessa indústria pode ser resumido da seguinte forma:

- Um caminhão refrigerado próprio é utilizado nas entregas em cozinhas industriais no Espírito Santo;

As entregas da linha de leite na região de Viçosa são feitas com dois caminhões térmicos do representante;

- Quatro caminhões refrigerados do representante são utilizados para distribuição das linhas de iogurtes, requeijão e manteiga em Viçosa, região de Ouro Preto, Ubá e Barbacena;

- Um caminhão do representante faz a entrega da linha de doce de leite em Viçosa;

- Um caminhão do distribuidor faz a entrega da linha de doce de leite na região metropolitana de Belo Horizonte;

- Caminhões da transportadora 1 são usados para a distribuição da linha de doces de leite em outras regiões e estados;

- Caminhões da transportadora 2 fazem entregas rápidas na cidade de São Paulo ou no estado do Rio de Janeiro; e

- Os pedidos feitos via internet são entregues através do serviço dos correios ou das transportadoras.

Como é possível notar, a logística de distribuição na indústria em questão é bastante definida. No 
entanto, com relação aos pedidos feitos via internet, foi identificado que, a cada novo pedido, o funcionário responsável pelo processamento dos pedidos analisa a quantidade solicitada em termos do peso total do pedido $(\mathrm{em} \mathrm{kg})$, o valor total da nota fiscal (em $\mathrm{R} \$$ ) e a distância entre a indústria e o cliente (em $\mathrm{km}$ ) e a urgência ou não do pedido para só então, com base nessas variáveis - e também na sua experiência -, decidir a melhor forma de distribuição desse pedido entre as três opções disponíveis (transportadora 1, transportadora 2 e correios).

Foi identificado que tanto a transportadora 1 quanto os correios atuam nos 26 estados brasileiros e Distrito Federal. Já a transportadora 2 atua nos quatro estados da região Sudeste e Bahia. Dessa forma, a amostra na qual se baseia a construção do modelo neste trabalho é composta pelos pedidos recebidos a partir dos estados do Sudeste, por se tratar de uma região onde as três opções disponíveis de distribuição atuam concomitantemente. Os vinte pedidos que compõem a amostra são apresentados na Tabela 1 e o tratamento numérico das variáveis mostradas nessa tabela resultou nos gráficos mostrados na Figura 2.

É interessante observar que as variáveis peso e valor da nota fiscal apresentam praticamente 0 mesmo comportamento. Isso é explicado pelo fato de uma variável ser diretamente proporcional à outra. 0 diagrama de dispersão apresentado na Figura 3 deixa esse fato mais claro ao indicar que 95,73\% da variação do valor da nota fiscal é explicado pela variação do peso.

Já que as variáveis peso e valor da nota fiscal apresentam praticamente o mesmo comportamento, uma delas pode deixar de ser usada como variável de entrada. Isso torna o modelo mais simples e reduz o esforço computacional da máquina. Desse modo, neste trabalho, o valor da nota fiscal será descartado como variável de entrada na construção do modelo.
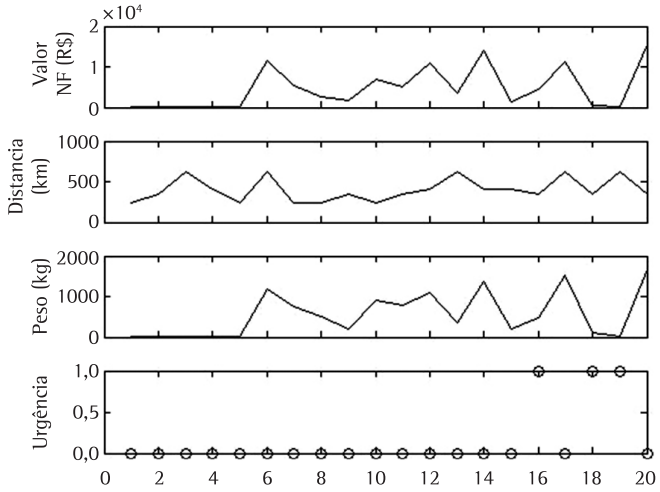

Figura 2. Gráfico das variáveis levantadas na amostra do problema.

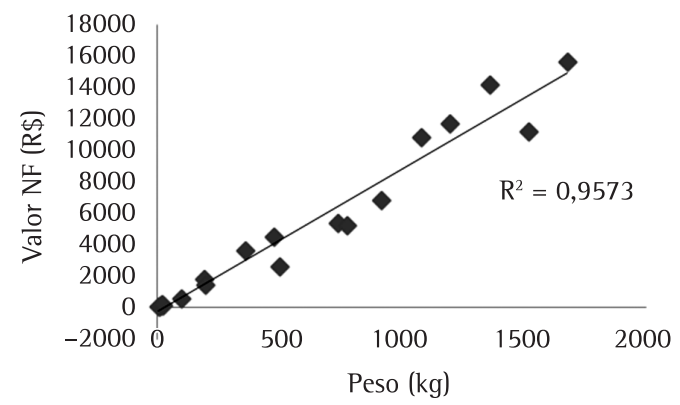

Figura 3. Diagrama de dispersão entre as variáveis peso e valor da nota fiscal.

Tabela 1. Vinte pedidos feitos via internet.

\begin{tabular}{|c|c|c|c|c|c|}
\hline Pedido & Distância (km) & Valor NF (R\$) & Peso (kg) & Urgência & Decisão \\
\hline 1 & 233 & 27,00 & 4,8 & & Correios \\
\hline 2 & 340 & 46,07 & 10 & & Correios \\
\hline 3 & 619 & 81,60 & 14,4 & & Correios \\
\hline 4 & 402 & 127,67 & 24,4 & & Correios \\
\hline 5 & 233 & 104,07 & 20 & & Correios \\
\hline 6 & 619 & $11.664,00$ & 1.200 & & Transportadora 1 \\
\hline 7 & 233 & $5.377,50$ & 740 & & Transportadora 1 \\
\hline 8 & 233 & $2.617,50$ & 500 & & Transportadora 1 \\
\hline 9 & 340 & $1.780,80$ & 192 & & Transportadora 1 \\
\hline 10 & 233 & $6.800,46$ & 917,6 & & Transportadora 1 \\
\hline 11 & 340 & $5.199,66$ & 778,4 & & Transportadora 1 \\
\hline 12 & 402 & $10.818,00$ & 1.080 & & Transportadora 1 \\
\hline 13 & 233 & $3.606,00$ & 360 & & Transportadora 1 \\
\hline 14 & 402 & $14.144,50$ & 1.360 & & Transportadora 1 \\
\hline 15 & 402 & $1.413,90$ & 196 & & Transportadora 1 \\
\hline 16 & 340 & $4.452,00$ & 480 & Sim & Transportadora 2 \\
\hline 17 & 619 & $11.186,40$ & 1.520 & & Transportadora 2 \\
\hline 18 & 340 & 523,50 & 100 & Sim & Transportadora 2 \\
\hline 19 & 619 & 199,44 & 19,20 & Sim & Transportadora 2 \\
\hline 20 & 340 & $15.582,00$ & 1.680 & & Transportadora 2 \\
\hline
\end{tabular}


A partir da experiência do funcionário da indústria e da análise dos gráficos da Figura 2, é possivel extrair as regras que servirão de base para o sistema de inferência fuzzy. Lembrando que os pedidos de 1 a 5 foram entregues via correios, os de 6 a 15, via transportadora 1 , e os de 16 a 20, via transportadora 2, as regras extraídas são as que seguem:

- Regra 1: Se o peso é pequeno, então a entrega via correios é muito provável;

- Regra 2: Se a distância é pequena e o peso não é pequeno então a entrega via transportadora 1 é muito provável;

- Regra 3: Se a distância é grande e o peso não é pequeno então a entrega via transportadora 1 é muito provável;

- Regra 4: Se a distância é média e o peso é médio e o pedido é não urgente, então a entrega via transportadora 1 é muito provável;

- Regra 5: Se a distância é muito grande e o peso é médio e o pedido é não urgente, então a entrega via transportadora 1 é muito provável;

- Regra 6: Se a distância é média e o pedido é urgente, então a entrega via transportadora 2 é muito provável;

- Regra 7: Se a distância é muito grande e o pedido é urgente, então a entrega via transportadora 2 é muito provável;

- Regra 8: Se a distância é média e o peso é grande e o pedido é não urgente, então a entrega via transportadora 2 é muito provável; e

- Regra 9: Se a distância é muito grande e o peso é grande e o pedido é não urgente, então a entrega via transportadora 2 é muito provável.

Desse modo, o modelo do sistema fuzzy de Mamdani criado para esse problema possui três variáveis de entrada, que são a distância, o peso e a urgência do pedido, e três saídas, que são correios, transportadora 1 e transportadora 2 .

A distância foi classificada em pequena, média, grande e muito grande e modelada por quatro funções de pertinência gaussianas (gaussmf). 0 domínio dessa variável vai de 100 a $700 \mathrm{~km}$ e os parâmetros das funções de pertinência são: pequena [50 233], média [50 340], grande [50 402] e muito grande [50 619].

0 peso foi classificado em pequeno, médio e grande e modelado por três funções de pertinência do tipo sino generalizada (gbellmff. 0 domínio dessa variável vai de 0 a $1.800 \mathrm{~kg}$ e os parâmetros das funções de pertinência são: pequeno [37,5 2,5 0], médio [450 2,5 900] e grande [450 2,5 1.800].

A urgência do pedido foi classificada em baixa, média e alta e modelada por três funções de pertinência gaussianas (gaussmf). 0 domínio dessa variável vai de 0 a $1 \mathrm{~kg}$ e os parâmetros das funções de pertinência são: baixa [0,2123 0], média [0,2123 0,5] e alta [0,2123 1]. É uma variável binária, onde o valor 0 indica um pedido não urgente, e 1 um pedido urgente.

A variável de saída correios foi classificada em pouco provável, provável e muito provável e modelada por três funções de pertinência triangulares (trimf). 0 domínio dessa variável vai de 0 a 1 e os parâmetros das funções de pertinência são: pouco provável [- $\left.\begin{array}{lll}-0,5 & 0 & 0,5\end{array}\right]$, provável $\left[\begin{array}{lll}0 & 0,5 & 1\end{array}\right]$ e muito provável $[0,5$ c 11 1,5].

Por fim, as variáveis de saída transportadora 1 e transportadora 2 foram classificadas em improvável, pouco provável, provável e muito provável e modeladas por quatro funções de pertinência gaussianas (gaussmff). 0 domínio dessas variáveis vai de 0 a 1 e os parâmetros das funções de pertinência são: improvável [0 0,1416], pouco provável [0,1416 0,3333], provável [0,1416 0,6667] e muito provável [0,1416 1].

Cada variável de saída fornecerá um valor entre 0 e 1 , onde o maior valor entre os três indica a melhor opção de envio para o pedido.

Por fim, as funções de inferência fuzzy e o método de defuzzificação foram ajustados da seguinte forma: and method (min), or method (max), implication (min), aggregation (max) e defuzzification (centroid).

Com o sistema de inferência fuzzy completamente definido e implementado na Fuzzy Logic Toolbox ${ }^{T M}$ do MATLAB $^{\circledR}$, é possível agora verificar o comportamento desse sistema através da comparação com os exemplos levantados. A decisão que o modelo apresenta quanto à forma de distribuição dos pedidos feitos via internet pode ser vista utilizando o Rule Viewer da Fuzzy Logic Toolbox ${ }^{\mathrm{TM}}$ (Figura 4).

Para o caso apresentado na Figura 4 tem-se que um pedido feito a partir de MG (distância de $233 \mathrm{~km}$ ), com um peso de $360 \mathrm{~kg}$ e não urgente, a melhor

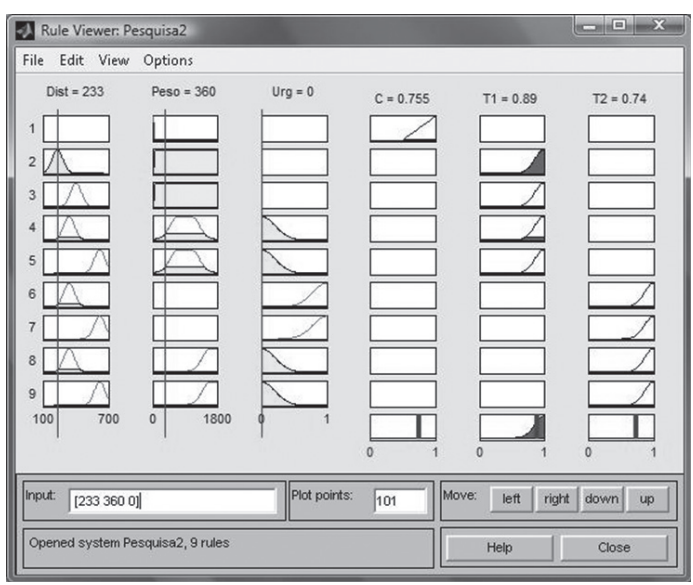

Figura 4. Decisão do sistema fuzzy de Mamdani. 
Tabela 2. Exemplos gerados para o processo de validação do modelo.

\begin{tabular}{|c|c|c|c|c|c|}
\hline Pedido & Distância (km) & Peso (kg) & Urgência & $\begin{array}{c}\text { Decisão } \\
\text { funcionário }\end{array}$ & $\begin{array}{l}\text { Decisão } \\
\text { modelo }\end{array}$ \\
\hline 1 & 233 & 10 & & Correios & Correios \\
\hline 2 & 619 & 1172 & Sim & Transportadora 2 & Transportadora 2 \\
\hline 3 & 402 & 1500 & & Transportadora 1 & Transportadora 1 \\
\hline 4 & 340 & 460 & & Transportadora 1 & Transportadora 1 \\
\hline 5 & 402 & 1334 & & Transportadora 1 & Transportadora 1 \\
\hline 6 & 340 & 1772 & Sim & Transportadora 2 & Transportadora 2 \\
\hline 7 & 233 & 9,6 & & Correios & Correios \\
\hline 8 & 619 & 312 & Sim & Transportadora 2 & Transportadora 2 \\
\hline 9 & 340 & 1229 & & Transportadora 1 & Transportadora 1 \\
\hline 10 & 619 & 894 & & Transportadora 1 & Transportadora 1 \\
\hline
\end{tabular}

decisão de entrega é via transportadora 1 (que apresentou um índice de 0,89 , o maior entre as três saídas). Esse caso é o mesmo do pedido 13 (Tabela 1), cuja decisão foi a mesma apresentada pelo modelo.

$\mathrm{Na}$ etapa de verificação desse modelo, repetiu-se o mesmo procedimento para os demais exemplos da Tabela 1, onde o modelo apresentou, em todos os casos, as mesmas decisões tomadas pelo especialista da indústria.

Para o processo de validação do modelo, foram gerados dez exemplos diferentes de pedidos feitos via internet. Esses exemplos foram fornecidos ao modelo e ao funcionário de logística da indústria. Os exemplos e as respostas fornecidas tanto pelo modelo quanto pelo funcionário são apresentados na Tabela 2.

\section{Conclusão}

A lógica fuzzy foi desenvolvida incialmente para modelagem de processos com variáveis contínuas. No entanto, os resultados deste trabalho mostram que a lógica fuzzy também produz resultados satisfatórios na modelagem problemas de natureza discreta. A partir dos dados levantados e dos resultados apresentados no processo de verificação do modelo, pode-se concluir que o sistema fuzzy foi transcrito de forma adequada, visto que o mesmo apresentou as mesmas decisões tomadas pelo especialista em logística em todos os vinte exemplos que compõem a amostra. Já os resultados apresentados na validação indicam que o modelo também foi coerente ao apresentar, nos dez exemplos gerados, as mesmas decisões tomadas pelo especialista em logística.

Entretanto, amostras maiores (tanto para verificação quanto validação) podem trazer resultados mais consistentes, aumentando o grau de confiabilidade dos resultados ainda que outras novas situações de teste apontem diferenças importantes entre as decisões fornecidas pelo modelo e o método heurístico.
Este trabalho buscou contribuir para a melhoria dos atuais métodos de processamento de pedidos e tomada de decisão logística, fornecendo um instrumento que traduz informações vagas, imprecisas e qualitativas, comuns na linguagem humana, em valores numéricos, tornando mais curto o tempo da preparação do pedido e possibilitando a tomada de ações procurando maximizar a eficiência da distribuição física.

Todavia, direcionar esforços para redução de custos de forma isolada pode não garantir o resultado desejado. É interessante planejar e coordenar ações gerenciais de forma integrada, avaliando todo o processo.

Com isso, o modelo proposto neste trabalho pode ser inserido em um sistema logístico mais amplo, composto, por exemplo, de mecanismos integrados de coleta e distribuição de pedidos eletrônicos, verificação de estoques e consolidação de cargas, podendo se tornar um importante fator de competitividade, já que pode conferir à indústria maior rapidez, confiabilidade e eficiência tanto no processamento quanto na distribuição dos pedidos.

\section{Referências}

BALlOU, R. H. Gerenciamento da Cadeia de Suprimentos - Logística Empresarial. 5. ed. Porto Alegre: Bookman, 2006. $616 \mathrm{p}$.

BOWERSOX, D. J.; ClOSS, D. J.; COOPER, M. B. Gestão Logística de Cadeia de Suprimentos. Porto Alegre: Bookman, 2006. 528 p.

CAMARGOS, F. L. Lógica Nebulosa: uma abordagem filosófica e aplicada. Florianópolis: UFSC, 2002. Disponivel em: <http://www.inf.ufsc.br/ barreto/ trabaluno/IAfernandolC.pdf>. Acesso em: 21 set. 2007.

CHOPRA, S.; MEINDL, P. Gerenciamento da Cadeia de Suprimentos. São Paulo: Prentice Hall, 2003. 465 p.

COUNCIL OF SUPPLY CHAIN MANAGEMENT PROFESSIONALS - CSCMP. Supply Chain - Logistics Definition. Disponível em: <http://cscmp.org/ AboutCSCMP/Definitions/Definitions.asp>. Acesso em: 01 out. 2007. 
FUJIMOTO, R. Y. Diagnóstico automático de defeitos em rolamentos baseado em Lógica Fuzzy. 2005. 158 f. Dissertação (Mestrado em Engenharia)-Escola Politécnica, Universidade de São Paulo, São Paulo, 2005.

GURGEL, F. A. Logística Empresarial. São Paulo: Atlas, 2000. $484 \mathrm{p}$.

OLIVEIRA JUNIOR, H. A. et al. (Coord.). Inteligência Computacional Aplicada à Administração, Economia e Engenharia em Matlab $^{\circledR}$. São Paulo: Thomson Learning, 2007. $370 \mathrm{p}$.

SANDRl, S.; CORREA, C. Lógica Nebulosa. São José dos Campos: ITA, 1999. Disponível em: <http://www.ele.ita. $\mathrm{br} / \mathrm{cnrn} /$ minicursos-5ern/log-neb.pdf>. Acesso em: 21 set. 2007.

SILVA, S. F. Identificação de Torque de Carga em motores de indução usando abordagem baseada em Sistemas Fuzzy. 2007. 95 f. Dissertação (Mestrado em Engenharia Elétrica)-Escola de Engenharia de São Carlos, Universidade de São Paulo, São Carlos, 2007.

THE MATHWORKS. Fuzzy Logic Toolbox ${ }^{T M}$ 2: User's Guide. Disponivel em: <http://www.mathworks.com/access/ helpdesk/help/pdf_doc/fuzzy/fuzzy.pdf>. Acesso em: 9 nov. 2008.

\begin{abstract}
This study focuses on the viability of fuzzy logic application to assist the management of a dairy industry in the process of decision-making in physical distribution logistics. The case study, carried out in a small dairy industry in the State of Minas Gerais, identified that, for each new order request via internet, the logistician copes with a large amount of information at the same time and decides, based on experience, the best way to distribute such orders. The purpose of this research was to present a fuzzy model as an alternative support to traditional methods for order processing and logistics decision-making. The results showed that the model adequately reproduced the problem proposed as it produced the same decisions made by the specialist and, if the weak points detected are observed, this model can be used as an important tool for the competitiveness of industries.
\end{abstract}

\title{
Study of physical distribution logistics of a dairy industry using fuzzy logic
}

\section{Keywords}

Physical distribution. Fuzzy logic. Dairy industry. 